\title{
new on the market
}

\section{Of mice and Tecnomice}

\section{A model for oestrogen receptor defects, and in vitro antibody production.}

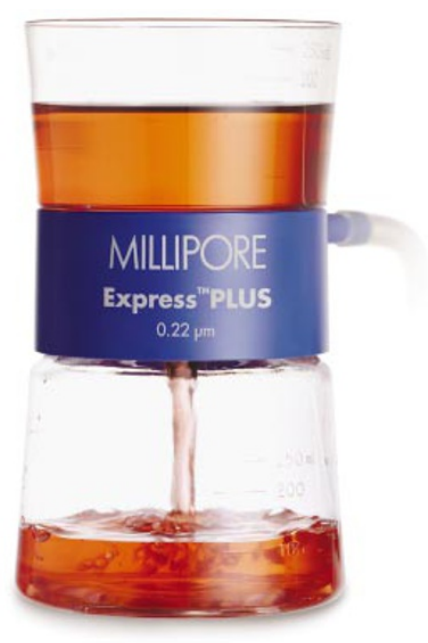

Up for the cup: Stericup goes Express.

ERKO- $\beta$

Taconic www.taconic.com

Fertile area of research

The Taconic ERKO- $\beta$ (oestrogen receptor- $\beta$ ) targeted mutation mouse model can be used to examine the role of oestrogen receptors in both reproductive and non-reproductive development. In this model, the esr2 gene, which encodes a protein of about $60 \mathrm{kDa}$, is disrupted in the embryonic stem cells, but esr1, the $\alpha$-oestrogen receptor, is not and remains functional. Both sexes of the homozygous ERKO- $\beta$ mouse develop normally and live to adulthood, but females experience infertility and have fewer offspring. The males have normal fertility, but suffer from prostatic and urinary bladder hyperplasia on ageing.

\section{Gel-Pro Analyzer 4.5}

Media Cybernetics www.mediacy.com Software that reads between the lines

Gel-Pro Analyzer is designed for molecular biologists needing to obtain images from electrophoretic gels, blots and colonies and extract qualitative and quantitative information from them. Gel-Pro eliminates the need for manual input by automating molecular weight and amount calculation through the use of pattern recognition of objects, lanes and bands. This upgrade to version 4.0 also adds support for GLP/CFR 21 Part 11 compliance.

\section{Express PLUS}

Millipore www.millipore.com Go with the flow

Millipore's Express PLUS membrane is now available in Stericup filter cups. These are fast- flowing, low-binding cups for sterilizing tissue culture media, buffers and other aqueous solutions. The membrane is claimed to be faster than other $0.2 \mu \mathrm{m}$ polyethersulphone membranes, with surface chemistry designed to ensure that key growth factors and proteins are not absorbed. The threads of the bottle attachment are slightly recessed to prevent contamination. The cups are available in sizes to process volumes from $150 \mathrm{ml}$ to 1 litre.

\section{siRNA products}

Dharmacon/Upstate www.upstate.com Silent partners

Upstate has teamed up with Dharmacon to offer a new line of gene-specific small interfering RNA (siRNA) duplexes paired with qualified antibodies (siAB). RNA interference is an increasingly important method for functional genetic analysis and target validation. Each siRNA/siAB pair consists of a Dharmacon siRNA SMARTpool reagent (5 nmoles) and a corresponding Upstate validated antibody. SMARTpools consist of four siRNA duplexes designed with Dharmacon's SMARTselection criteria against the target gene. siRNA SMARTpools are transfection-ready and are suitable to use for human gene knockdown.

\section{Tecnomouse}

Integra Biosciences

www.integra-biosciences.com

In vitro antibody production

Tecnomouse makes it possible to cultivate cells in vitro in a reliable and economic manner and to harvest the products over a period of months, minimizing the need for lab animals. The modular system is based on hollow-fibre membranes incorporated in a bioreactor known as the CultureCassette. Here, cell densities similar to those in tissue are produced. The compartmentalized design of the CultureCassette enables both $\mathrm{O}_{2} / \mathrm{CO}_{2}$ and media supply to be controlled and adapted to the specific needs of the cells.

These notes are compiled in the Nature office from information provided by the manufacturers. 